\title{
ZERO DISTRIBUTION OF COMPOSITE POLYNOMIALS AND POLYNOMIALS BIORTHOGONAL TO EXPONENTIALS
}

\author{
D. S. LUBINSKY ${ }^{1}$ AND A. SIDI ${ }^{2}$
}

Abstract. We analyze polynomials $P_{n}$ that are biorthogonal to exponentials $\left\{e^{-\sigma_{n, j} x}\right\}_{j=1}^{n}$, in the sense that

$$
\int_{0}^{\infty} P_{n}(x) e^{-\sigma_{n, j} x} x^{\alpha} d x=0,1 \leq j \leq n .
$$

Here $\alpha>-1$. We show that the zero distribution of $P_{n}$ as $n \rightarrow \infty$ is closely related to that of the associated exponent polynomial

$$
Q_{n}(y)=\prod_{j=1}^{n}\left(y+1 / \sigma_{n, j}\right)=\sum_{j=0}^{n} q_{n, j} y^{j} .
$$

More precisely, we show that the zero counting measures of $\left\{P_{n}(-4 n x)\right\}_{n=1}^{\infty}$ converge weakly iff the zero counting measures of $\left\{Q_{n}\right\}_{n=1}^{\infty}$ converge weakly. A key step is relating the zero distribution of such a polynomial to that of the composite polynomial

$$
\sum_{j=0}^{n} q_{n, j} \Delta_{n, j} x^{j}
$$

under appropriate assumptions on $\left\{\Delta_{n, j}\right\}$.

Research supported by NSF grant DMS0400446 and US-Israel BSF grant 2004353 Keywords: Biorthogonal polynomials, zero distribution, Laguerre polynomials. AMS Classification: 42C05, 30C15, 41A99

\section{Polynomials Biorthogonal to exponentials}

Let $\alpha>-1$ and $\left\{\sigma_{n, j}\right\}_{j=1}^{n}$ be distinct exponents in $(0, \infty)$. Then we may determine a monic polynomial $P_{n}$ of degree $n$ by the biorthogonality conditions

$$
\int_{0}^{\infty} P_{n}(x) e^{-\sigma_{n, j} x} x^{\alpha} d x=0,1 \leq j \leq n .
$$

In developing methods for convergence acceleration, and numerical integration of singular integrands, the second author introduced some classes of polynomials of this type [15], [16], [17], [18]. These include polynomials $P_{n}$ that correspond to the following choices of exponents:

(I) $\sigma_{n, j}=j+\beta, 1 \leq j \leq n$, some $\beta>-1$;

(II) $\left\{\sigma_{n, j}^{-1}\right\}_{j=1}^{n}$ are the zeros of Sidi polynomials, which will be discussed in Example 1 of Section 2;

(III) $\left\{\sigma_{n, j}^{-1}\right\}_{j=1}^{n}$ are the zeros of Legendre polynomials scaled to $(0,1)$. We shall discuss these special choices in greater detail in Section 2.

The first case arises from polynomials that are, in a certain sense, dual to the Sidi polynomials. The latter arise in numerical quadrature of integrals of the form

Date: September 18, 2007. 
$\int_{0}^{1} f(x) w(x) d x$, where the weight $w$ has an algebraic or logarithmic singularity at 0 or 1.

In the last two cases, the polynomial $P_{n}$ arises as the denominator of a rational approximation to $H(z)=\int_{0}^{\infty} \frac{w(x)}{x-z} d x$, where $w$ is an appropriate weight function. The rational approximation itself is determined by applying various convergence acceleration methods to the asymptotic expansion as $z \rightarrow \infty$ of $H(z)$, and its poles and residues are respectively the abscissas and weights in the quadrature rule. The resulting quadrature rules are especially useful in approximation of integrals of the form $\int_{0}^{\infty} f(x) w(x) d x$.

Apart from their practical use, the polynomials $\left\{P_{n}\right\}$ clearly have intrinsic interest. Indeed, there is a growing body of research that deals with polynomials that possess some generalized type of orthogonality. The term "biorthogonality" is often used to describe this, although these are usually different from the classical theory of biorthogonal polynomials, which involves two different sequences of polynomials. In much of the research of recent decades, the second sequence of polynomials is replaced by a sequence of functions that need not be polynomials at all. For example, the Sidi polynomials $D_{n}^{(0,0)}$ are determined by the biorthogonality relation

$$
\int_{0}^{1} D_{n}^{(0,0)}(x)(\log x)^{j} d x=0,0 \leq j<n .
$$

Their properties and generalizations have been studied in [9], [10], [11], [15], [18].

An elegant and general theory of biorthogonal polynomials was developed by Iserles and Norsett [5]. They considered the more general situation

$$
\int P_{n}(x) d \phi\left(x, \sigma_{j}\right)=0,1 \leq j \leq n .
$$

Under appropriate conditions on the measure $\phi$, they obtained existence and uniqueness of $P_{n}$, Rodrigues-type formulae, simplicity of the zeros, interlacing properties, and explicit representations. Their original motivation came from numerical solution of differential equations, but the methods and results also were useful in analysing how various transformations map polynomials with zeros in one given region, into polynomials with zeros in another given region [6], [7]. Still more general biorthogonal polynomials were investigated by Brezinski in the monograph [3]. We emphasize that this list is incomplete. Biorthogonal polynomials in continuous or discrete settings have been studied by many authors, though they typically have a different flavor from that we consider here.

It turns out that $P_{n}$ of (1.1) is closely related to the monic polynomial

$$
Q_{n}(y)=\prod_{j=1}^{n}\left(y+\sigma_{n, j}^{-1}\right)=\sum_{j=0}^{n} q_{n, j} y^{j} .
$$

We call $Q_{n}$ the associated exponent polynomial for $P_{n}$.

One of our main tools is a simple relationship between $P_{n}$ and $Q_{n}$ involving a certain type of composition of polynomials. Given

$$
R(x)=\sum_{j=0}^{n} r_{j} x^{j} \text { and } S(x)=\sum_{j=0}^{n} s_{j} x^{j},
$$


we define their composition

$$
(R \# S)(x)=\sum_{j=0}^{n} \frac{r_{j} s_{j}}{\left(\begin{array}{c}
n \\
j
\end{array}\right)} x^{j} .
$$

This operation arises in a classical theorem of Szegó on zeros of composite polynomials. See for example [12, p. 65], [13, p. 191]. We also need the explicit representation for the Laguerre polynomials

$$
L_{n}^{(\alpha)}(x)=\frac{1}{n !} \sum_{j=0}^{n}\left(\begin{array}{l}
n \\
j
\end{array}\right) \frac{\Gamma(\alpha+n+1)}{\Gamma(\alpha+j+1)}(-x)^{j}=\frac{(-1)^{n}}{n !} x^{n}+\ldots,
$$

see [14, p. 201], [22, p. 101], where equivalent formulae are presented.

\section{Proposition 1.1}

Let $\alpha>-1,\left\{\sigma_{n, j}\right\}_{j=1}^{n}$ be $n$ distinct positive numbers, and $P_{n}$ be a monic polynomial of degree $n$ determined by the relations (1.1), and $Q_{n}$ be given by (1.2). Then $P_{n}$ exists, is unique, and is given by

$$
P_{n}(x)=(-1)^{n} n !\left(Q_{n} \# L_{n}^{(\alpha)}\right)(x)=\sum_{j=0}^{n}(-1)^{n-j} q_{n, j} \frac{\Gamma(\alpha+n+1)}{\Gamma(\alpha+j+1)} x^{j} .
$$

Moreover, $P_{n}$ has $n$ simple zeros in $(0, \infty)$.

Proof

Observe that if $P_{n}$ is given by the last expression in the right-hand side of (1.6),

$$
\begin{aligned}
\frac{(-1)^{n}}{\Gamma(\alpha+n+1)} \int_{0}^{\infty} P_{n}(x) e^{-\sigma_{n, k} x} x^{\alpha} d x & =\sum_{j=0}^{n} \frac{q_{n, j}}{\Gamma(\alpha+j+1)}(-1)^{j} \int_{0}^{\infty} x^{\alpha+j} e^{-\sigma_{n, k} x} d x \\
& =\sigma_{n, k}^{-\alpha-1} \sum_{j=0}^{n} q_{n, j}\left(-\sigma_{n, k}\right)^{-j} \\
& =\sigma_{n, k}^{-\alpha-1} Q_{n}\left(-\sigma_{n, k}^{-1}\right)=0 .
\end{aligned}
$$

This gives the orthogonality relations, as well as establishing the existence of $P_{n}$ of course. The first identity in (1.6) then also follows. Uniqueness of $P_{n}$ follows from the fact that $\left\{e^{-\sigma_{n, j} x}\right\}_{j=1}^{n}$ is a Chebyshev system. Indeed, suppose $P_{n}^{*}$ is a different such monic polynomial. We can find a linear combination $h(x)$ of $\left\{e^{-\sigma_{n, j} x}\right\}_{j=1}^{n}$ changing sign exactly at the at most $n-1$ sign changes of $P_{n}-P_{n}^{*}$ in $(0, \infty)$. Then

$$
0=\int_{0}^{\infty} x^{\alpha}\left(P_{n}-P_{n}^{*}\right)(x) h(x) d x
$$

which is impossible as the integrand is positive except at at most $n-1$ points. A similar argument shows that $P_{n}$ has $n$ simple zeros in $(0, \infty)$.

Let $\nu_{n}$ denote the zero counting function for $Q_{n}$, so that

$$
\nu_{n}[a, b]=\frac{1}{n}\left(\text { Number of zeros of } Q_{n} \text { in }[a, b]\right) .
$$

Equivalently,

$$
\nu_{n}=\frac{1}{n} \sum_{j=1}^{n} \delta_{-1 / \sigma_{n, j}}
$$


where $\delta_{a}$ denotes a unit mass at $a$. We also let $\mu_{n}$ denote the zero counting function of $P_{n}(-4 n x)$, so that

$$
\left.\mu_{n}[a, b]=\frac{1}{n} \text { (Number of zeros of } P_{n}(-4 n x) \text { in }[a, b]\right) .
$$

Note that we are effectively contracting the zeros of $P_{n}$, and if we denote these by $\left\{x_{n, j}\right\}_{j=1}^{n}$,

$$
\mu_{n}=\frac{1}{n} \sum_{j=1}^{n} \delta_{-x_{n, j} / 4 n} .
$$

Recall the definition of weak convergence: we write

$$
\nu_{n} \stackrel{*}{\longrightarrow} \nu, n \rightarrow \infty,
$$

if for every continuous function $f$ with compact support,

$$
\lim _{n \rightarrow \infty} \int f d \nu_{n}=\int f d \nu
$$

Our main result is

\section{Theorem 1.2}

Assume that for some $B>0$ and all $n \geq 1$, we are given distinct exponents $\left\{\sigma_{n, j}\right\}_{j=1}^{n}$ in $[B, \infty)$. Let $\left\{P_{n}\right\}$ denote the corresponding monic biorthogonal polynomials, and $\left\{Q_{n}\right\}$ the associated exponent polynomials. Let $\left\{\nu_{n}\right\}$ denote the zero counting measures of $\left\{Q_{n}\right\}$, and let $\left\{\mu_{n}\right\}$ denote the zero counting measures of $\left\{P_{n}(-4 n x)\right\}$. The following are equivalent:

(a) There exists a measure $\nu$ such that

$$
\nu_{n} \stackrel{*}{\longrightarrow} \nu, n \rightarrow \infty .
$$

(b) There exists a measure $\mu$ such that

$$
\mu_{n} \stackrel{*}{\longrightarrow} \mu, n \rightarrow \infty .
$$

Moreover, assuming the weak convergence, both $\mu$ and $\nu$ have support in $[-1 / B, 0]$, and $\mu$ will have a point mass at 0 of size $\lambda$ iff $\nu$ does. Uniformly for $z$ in compact subsets of $\mathbb{C} \backslash[-1 / B, 0]$,

$$
\lim _{n \rightarrow \infty}\left|P_{n}(-4 n z)\right|^{1 / n} /(4 n)=\exp \left(\int_{-1 / B}^{0} \log |z-t| d \mu(t)\right) .
$$

Thus when the zero counting measures of $\left\{Q_{n}\right\}$ converge weakly, to a measure without a mass point at 0 , then the same is true of the zero counting measures for $\left\{P_{n}(-4 n x)\right\}$. More information is presented in the following theorems, proved using an extension of a classic result of Van Assche, Fano and Ortolani [1]. They are necessarily complicated to formulate, because the mass point at 0 forces us to distinguish between lower order, and higher order, coefficients of $\left\{P_{n}\right\}$.

\section{Theorem 1.3}

Assume the hypotheses of Theorem 1.2 and that $\nu_{n} \stackrel{*}{\longrightarrow} \nu, n \rightarrow \infty$, where $\nu$ has a point mass at 0 of size $\lambda \in[0,1]$. Write

$$
P_{n}(-4 n z) /(-4 n)^{n}=\sum_{k=0}^{n} p_{n, k} z^{k}
$$


(I) Assume $\lambda>0$ and let $0<\lambda^{\prime}<\lambda$. Then as $n \rightarrow \infty$,

$$
\begin{gathered}
\max _{k \leq \lambda^{\prime} n} \frac{1}{n} \log q_{n, k} \rightarrow-\infty ; \\
\max _{k \leq \lambda^{\prime} n} \frac{q_{n, k}}{q_{n, k+1}} \rightarrow 0 ; \\
\max _{k \leq \lambda^{\prime} n} \frac{1}{n} \log p_{n, k} \rightarrow-\infty ;
\end{gathered}
$$

and

$$
\max _{k \leq \lambda^{\prime} n} \frac{p_{n, k}}{p_{n, k+1}} \rightarrow 0
$$

(II) Assume $\lambda<1$.

(a) There is a concave and differentiable function $g:(0,1) \rightarrow \mathbb{R}$, and a function $f$ defined by $f=e^{g^{\prime}}$ with the following properties: as $k, n \rightarrow \infty$ with

$$
k / n \rightarrow 1-(1-\lambda) d \in(\lambda, 1),
$$

we have

$$
\begin{gathered}
\frac{1}{n(1-\lambda)} \log q_{n, k} \rightarrow g(d) ; \\
\frac{q_{n, k}}{q_{n, k+1}} \rightarrow e^{g^{\prime}(d)}=f(d) .
\end{gathered}
$$

Let

$G(d)=g(d)-d \ln 4-\frac{1}{1-\lambda}\{(1-(1-\lambda) d) \log (1-(1-\lambda) d)-(1-(1-\lambda) d)\} ;$

$$
F(d)=\frac{1-(1-\lambda) d}{4} f(d)
$$

Then as $k, n \rightarrow \infty$ with (1.15) holding, we have

$$
\begin{gathered}
\frac{1}{n(1-\lambda)} \log p_{n, k} \rightarrow G(d) \\
\frac{p_{n, k}}{p_{n, k+1}} \rightarrow F(d) .
\end{gathered}
$$

Concerning the measures $\nu$ and $\mu$, we prove:

\section{Theorem 1.4}

Assume the hypotheses and notation of Theorem 1.3 and that $\lambda<1$. The functions $f$ and $F$ are strictly decreasing functions on $(0,1)$ with ranges $(0, \infty)$. Their inverses $f^{-1}$ and $F^{-1}$ are related to

$$
\nu^{*}=\frac{\nu-\lambda \delta_{0}}{1-\lambda} \text { and } \mu^{*}=\frac{\mu-\lambda \delta_{0}}{1-\lambda}
$$

by

$$
f^{-1}(x)=\int_{-1 / B}^{0} \frac{y}{y-x} d \nu^{*}(y), x \in(0, \infty) .
$$




$$
F^{-1}(x)=\int_{-1 / B}^{0} \frac{y}{y-x} d \mu^{*}(y), x \in(0, \infty) .
$$

We close this section with some more representations of $P_{n}$. Recall the confluent hypergeometric function

$$
{ }_{1} F_{1}(1 ; \alpha+1 ; z)=\sum_{j=0}^{\infty} \frac{z^{j}}{(1+\alpha)_{j}},
$$

where $(a)_{n}=a(a+1) \ldots(a+n-1)$ denotes the Pochhammer symbol.

\section{Theorem 1.5}

Let $\alpha>-1,\left\{\sigma_{n, j}\right\}_{j=1}^{n}$ be $n$ distinct positive numbers, and let $P_{n}$ be the monic polynomial of degree $n$ determined by the relations (1.1), and $Q_{n}$ be given by (1.2). Then

$$
P_{n}(x)=(-1)^{n} \frac{\Gamma(\alpha+n+1)}{\Gamma(\alpha+1)} \frac{1}{2 \pi i} \int_{|t|=1} \frac{Q_{n}(t)}{t}{ }_{1} F_{1}\left(1 ; \alpha+1 ;-\frac{x}{t}\right) d t .
$$

$$
Q_{n}(y)=\frac{(-1)^{n}}{\Gamma(\alpha+n+1)} \int_{0}^{\infty} e^{-t} t^{\alpha} P_{n}(-y t) d t
$$

$$
x^{\alpha} P_{n}(x)=\frac{(-1)^{n} \Gamma(\alpha+n+1)}{2 \pi i} \int_{\gamma-i \infty}^{\gamma+i \infty} e^{s x} s^{-\alpha-1} Q_{n}\left(-s^{-1}\right) d s,
$$

where $\gamma>0$ and the contour of integration is the line $\operatorname{Re} s=\gamma$.

(IV) Abbreviate $\sigma_{n, j}$ as $\sigma_{j}, 1 \leq j \leq n$. Then

$$
\frac{\operatorname{Pet}\left[\begin{array}{ccccc}
1 & \sigma_{1}^{-1} & \sigma_{1}^{-2} & \cdots & \sigma_{1}^{-n} \\
1 & \sigma_{2}^{-1} & \sigma_{2}^{-2} & \cdots & \sigma_{2}^{-n} \\
\vdots & \vdots & \vdots & \ddots & \vdots \\
1 & \sigma_{n}^{-1} & \sigma_{n}^{-2} & \cdots & \sigma_{n}^{-n} \\
\frac{1}{\Gamma(\alpha+1)} & \frac{x}{\Gamma(\alpha+2)} & \frac{x^{2}}{\Gamma(\alpha+3)} & \cdots & \frac{x^{n}}{\Gamma(\alpha+n+1)}
\end{array}\right]}{\operatorname{det}\left[\begin{array}{ccccc}
1 & \sigma_{1}^{-1} & \sigma_{1}^{-2} & \cdots & \sigma_{1}^{-n+1} \\
1 & \sigma_{2}^{-1} & \sigma_{2}^{-2} & \cdots & \sigma_{2}^{-n+1} \\
\vdots & \vdots & \vdots & \ddots & \vdots \\
1 & \sigma_{n}^{-1} & \sigma_{n}^{-2} & \cdots & \sigma_{n}^{-n+1}
\end{array}\right]} .
$$

This paper is organized as follows. In Section 2, we discuss some examples. In Section 3, we state an extension of a result of Van Assche, Fano and Ortolani, and a consequence for zero distribution of composite polynomials. In Section 4 , we prove the results of Section 3. In Section 5, we prove Theorems 1.2 through Theorem 1.5, and the corollaries of Section 2. 


\section{EXAmples}

\section{Example 1}

In developing methods for convergence acceleration, and numerical integration of singular integrands, the second author introduced [15], [18] the Sidi polynomials

$$
D_{n}^{(\alpha, \beta)}(t)=\sum_{j=0}^{n}(-1)^{n-j}\left(\begin{array}{l}
n \\
j
\end{array}\right)(\beta+j+1)^{\alpha+n} t^{j} .
$$

Here $\alpha, \beta>-1$. When $\alpha$ is a non-negative integer, they admit the Rodrigues-type representation

$$
D_{n}^{(\alpha, \beta)}(t)=(-1)^{n} t^{-\beta-1}\left(t \frac{d}{d t}\right)^{\alpha+n}\left[t^{\beta+1}(1-t)^{n}\right] .
$$

For all $\alpha, \beta>-1$, they satisfy the biorthogonality relation

$$
\int_{0}^{1} D_{n}^{(\alpha, \beta)}(t)\left(\log t^{-1}\right)^{\alpha+k} t^{\beta} d t=0,0 \leq k \leq n-1 .
$$

In the special case $\alpha=\beta=0$, the authors established the asymptotic behavior as $n \rightarrow \infty$ of $D_{n}^{(0,0)}$ by steepest descent, and deduced their zero distribution [9]. This inspired the first author to consider with H. Stahl and I. Soran, biorthogonality relations where $\log t^{-1}$ is replaced by a positive, possibly non-integer power of $t$ $[10],[11]$.

The biorthogonality relation (2.2) leads to dual monic polynomials $\Lambda_{n}^{(\alpha, \beta)}$ satisfying the relations $[18$, p. 368]

$$
\int_{0}^{1} D_{m}^{(\alpha, \beta)}(t) \Lambda_{n}^{(\alpha, \beta)}\left(\log t^{-1}\right) t^{\beta}\left(\log t^{-1}\right)^{\alpha} d t=0 \text { if } m \neq n .
$$

Equivalently,

or

$$
\int_{0}^{1} \Lambda_{n}^{(\alpha, \beta)}\left(\log t^{-1}\right) t^{\beta+k}\left(\log t^{-1}\right)^{\alpha} d t=0,0 \leq k \leq n-1
$$

$$
\int_{0}^{\infty} \Lambda_{n}^{(\alpha, \beta)}(x) e^{-(k+\beta+1) x} x^{\alpha} d x=0,0 \leq k \leq n-1 .
$$

Thus $\Lambda_{n}^{(\alpha, \beta)}(x)$ is orthogonal to the system of exponentials $\left\{e^{-(k+\beta) x}\right\}_{k=1}^{n}$ with weight $x^{\alpha}$. Observe that for $P_{n}=\Lambda_{n}^{(\alpha, \beta)}$, we have

$$
Q_{n}(y)=\prod_{k=1}^{n}\left(y+\frac{1}{k+\beta}\right)
$$

and hence the zero counting measures $\left\{\nu_{n}\right\}$ converge weakly to a unit mass at 0 :

$$
\nu_{n} \stackrel{*}{\rightarrow} \delta_{0} \text { as } n \rightarrow \infty \text {. }
$$

Then Theorems 1.2-1.4 and the aforementioned theorem of Szegö on composite polynomials give: 


\section{Corollary 2.1}

(a) All zeros of $\Lambda_{n}^{(\alpha, \beta)}$ lie in the interval $\left(0,\left(4 n+2 \alpha+2 \frac{1}{2}\right) /(1+\beta)\right)$.

(b) The zero counting measures $\left\{\mu_{n}\right\}$ of $\left\{\Lambda_{n}^{(\alpha, \beta)}(-4 n x)\right\}$ satisfy

$$
\mu_{n} \stackrel{*}{\rightarrow} \delta_{0} \text { as } n \rightarrow \infty \text {. }
$$

Moreover, uniformly for $z$ in compact subsets of $\mathbb{C} \backslash[-1,0]$,

$$
\lim _{n \rightarrow \infty}\left|\Lambda_{n}^{(\alpha, \beta)}(-4 n z)\right|^{1 / n} /(4 n)=|z| .
$$

It would be instructive to investigate the behavior of $\Lambda_{n}^{(\alpha, \beta)}$ under different scalings.

\section{Example 2}

In the same context of numerical integration and convergence acceleration, the second author introduced [16] the polynomials

$$
G_{n}^{(\alpha)}(x)=\sum_{j=0}^{n}(-1)^{n-j}\left(\begin{array}{c}
n \\
j
\end{array}\right) \frac{(j+1)^{n}}{\Gamma(j+\alpha+1)} x^{j} .
$$

These admit the Rodrigues type formula

$$
G_{n}^{(\alpha)}(x)=C_{n} \frac{1}{x}\left(x \frac{d}{d x}\right)^{n}\left[x L_{n}^{(\alpha)}(x)\right],
$$

where $C_{n}$ is an appropriate constant, and the biorthogonality relation [17]

$$
\int_{0}^{\infty} G_{n}^{(\alpha)}(x) e^{-\sigma_{n, k} x} x^{\alpha} d x=0,1 \leq k \leq n,
$$

where $\left\{\sigma_{n, k}\right\}_{k=1}^{n}$ are the reciprocals of the roots of the polynomials $D_{n}^{(0,0)}$ above, that is,

$$
D_{n}^{(0,0)}\left(\sigma_{n, k}^{-1}\right)=0,1 \leq k \leq n .
$$

Thus in this case $Q_{n}(y)$ is a constant multiple of $D_{n}^{(0,0)}(-y)$. It is known [9, Theorem 1.3, p. 345] that the zero counting measure $\nu_{n}$ of $Q_{n}(y)=C D_{n}^{(0,0)}(-y)$ converges weakly to an absolutely continuous measure $\nu$, which may be defined by

$$
\nu^{\prime}(-x)=-\frac{1}{\pi h^{\prime}\left(h^{[-1]}(x)\right)}, \quad x \in(0,1),
$$

where $h^{[-1]}$ is the inverse of the function

$$
h(y)=\frac{\sin y}{y} e^{y \cot y-1}, y \in[0, \pi] .
$$

It is shown there that

$$
\begin{aligned}
& \nu^{\prime}(x)=\frac{1}{\pi \sqrt{2(1+x)}}(1+o(1)), \quad x \rightarrow(-1)+; \\
& \nu^{\prime}(x)=\frac{1}{\left.|x||\log | x\right|^{2}}(1+o(1)), \quad x \rightarrow 0-.
\end{aligned}
$$

\section{Corollary $\mathbf{2 . 2}$}

(a) All zeros of $G_{n}^{(\alpha)}$ lie in the interval $\left(0,4 n+2 \alpha+2 \frac{1}{2}\right)$. 
(b) The zero counting measures $\left\{\mu_{n}\right\}$ of $\left\{G_{n}^{(\alpha)}(-4 n x)\right\}$ converge weakly to a measure $\mu$ with support in $[-1,0]$, and without a mass point at 0 , satisfying (1.23), where

$$
F(x)=\frac{(1-x)^{2}}{4 x} \exp \left(-\frac{1}{1-x}\right), x \in(0,1)
$$

\section{Example 3}

A third class of polynomials with a biorthogonality relation that arises in numerical integration is [20]

$$
M_{n}^{(\alpha)}(x)=\sum_{j=0}^{n}(-1)^{n-j}\left(\begin{array}{l}
n \\
j
\end{array}\right) \frac{(j+1)_{n}}{\Gamma(j+\alpha+1)} x^{j} .
$$

Its Rodrigues type formula is

$$
M_{n}^{(\alpha)}(x)=C_{n}\left(\frac{d}{d x}\right)^{n}\left[x^{n} L_{n}^{(\alpha)}(x)\right],
$$

where again $C_{n}$ is an appropriate constant. Here the biorthogonality relation is

$$
\int_{0}^{\infty} M_{n}^{(\alpha)}(x) e^{-\sigma_{n, k} x} x^{\alpha} d x=0,1 \leq k \leq n,
$$

where $\left\{\sigma_{n, k}^{-1}\right\}_{k=1}^{n}$ are the roots of the Legendre polynomial for $[0,1]$. That is,

$$
Q_{n}\left(-\sigma_{n, k}^{-1}\right)=0,1 \leq k \leq n,
$$

where for a suitable constant $C$,

$$
Q_{n}(-y)=C\left(\frac{d}{d y}\right)^{n}[y(1-y)]^{n} .
$$

\section{Corollary 2.3}

(a) All zeros of $M_{n}^{(\alpha)}$ lie in the interval $\left(0,4 n+2 \alpha+2 \frac{1}{2}\right)$.

(b) The zero counting measures $\left\{\mu_{n}\right\}$ of $\left\{M_{n}^{(\alpha)}(-4 n x)\right\}$ converge weakly to a measure $\mu$ with support in $[-1,0]$, and without a mass point at 0 , satisfying (1.23), where

$$
F(x)=\frac{(1-x)^{3}}{4 x(2-x)}, x \in(0,1) .
$$

\section{Composite Polynomials}

We begin with a statement of a result of an extension of a classic result of Van Assche, Fano and Ortolani [1]. They considered the case where the measure $\nu$ has no mass point at 0 .

\section{Theorem 3.1}

Let $A>0$. For $n \geq 1$, let

$$
V_{n}(x)=\prod_{j=1}^{n}\left(x-\nu_{n, j}\right)=\sum_{j=0}^{n} v_{n, j} x^{j},
$$


where all $\nu_{n, j} \in[-A, 0)$. Assume that the zero counting measures $\left\{\nu_{n}\right\}$ of $\left\{V_{n}\right\}$ satisfy

$$
\nu_{n} \stackrel{*}{\rightarrow} \nu \text { as } n \rightarrow \infty
$$

where $\nu$ has a point mass of size $\lambda$ at 0 .

(I) Assume $\lambda>0$ and let $0<\lambda^{\prime}<\lambda$. Then as $n \rightarrow \infty$,

$$
\begin{gathered}
\max _{k \leq \lambda^{\prime} n} \frac{1}{n} \log v_{n, k} \rightarrow-\infty ; \\
\max _{k \leq \lambda^{\prime} n} \frac{v_{n, k}}{v_{n, k+1}} \rightarrow 0 .
\end{gathered}
$$

(II) Assume $\lambda<1$.

(a) Then there is a concave and differentiable function $g:(0,1) \rightarrow \mathbb{R}$, and a function $f$ defined by $f=e^{g^{\prime}}$ with the following properties: as $k, n \rightarrow \infty$ with

$$
\frac{k}{n} \rightarrow 1-(1-\lambda) d \in(\lambda, 1)
$$

we have

$$
\begin{gathered}
\frac{1}{n(1-\lambda)} \log v_{n, k} \rightarrow g(d) ; \\
\frac{v_{n, k}}{v_{n, k+1}} \rightarrow e^{g^{\prime}(d)}=f(d) .
\end{gathered}
$$

(III) Assume $\lambda<1$ and let

$$
\nu^{*}=\frac{\nu-\lambda \delta_{0}}{1-\lambda} .
$$

The function $f$ is strictly decreasing and continuous on $(0,1)$ with range $(0, \infty)$. Its inverse $f^{-1}$ is related to $\nu^{*}$ by

$$
f^{-1}(x)=\int_{-A}^{0} \frac{y}{y-x} d \nu^{*}(y), x \in(0, \infty) .
$$

Moreover,

$$
g(d)=-(1-d) \log f(d)+\int_{-A}^{0} \log [f(d)-y] d \nu^{*}(y), d \in(0,1) .
$$

All the assertions of this theorem go through if we assume the hypotheses holds only for some subsequence $\left\{V_{n}\right\}_{n \in S}$, provided all the limits are then restricted to that subsequence.

We also used $\nu_{n}$ for the zero counting measure of $Q_{n}$, but expect that no confusion should arise - especially as we shall deduce Theorem 1.2 by taking $V_{n}=Q_{n}$. From this result, we shall deduce a result on zero distribution of composite polynomials:

\section{Theorem 3.2}

Let $A \geq 1$. For $n \geq 1$, let

$$
W_{n}(x)=\prod_{j=1}^{n}\left(x-\omega_{n, j}\right)=\sum_{j=0}^{n} w_{n, j} x^{j},
$$


where all $\omega_{n, j} \in[-A, 0)$. Assume that $\left\{V_{n}\right\}$ are as in Theorem 3.1 and the coeffcients of $V_{n}$ and $W_{n}$ are connected by the relation

$$
w_{n, k}=v_{n, k} \Delta_{n, k},
$$

where as $n, k \rightarrow \infty$ with (3.3) holding, we have

$$
\frac{1}{n(1-\lambda)} \log \Delta_{n, k} \rightarrow h(d) \text {. }
$$

Moreover, we assume that if $\eta \in\left(0, \frac{1}{2}\right)$, there exists $C>1$ such that whenever $k / n \in(\eta, 1-\eta)$

$$
C^{-1}<\frac{\Delta_{n, k}}{\Delta_{n, k+1}}<C
$$

Then

(I) The zero counting measures $\left\{\omega_{n}\right\}$ of $\left\{W_{n}\right\}$ satisfy

$$
\omega_{n} \stackrel{*}{\rightarrow} \omega \text { as } n \rightarrow \infty,
$$

where $\omega$ has a point mass of size $\lambda$ at 0 .

(II) Assume $\lambda>0$ and let $0<\lambda^{\prime}<\lambda$. Then as $n \rightarrow \infty$,

$$
\begin{gathered}
\max _{k \leq \lambda^{\prime} n} \frac{1}{n} \log w_{n, k} \rightarrow-\infty ; \\
\max _{k \leq \lambda^{\prime} n} \frac{w_{n, k}}{w_{n, k+1}} \rightarrow 0 .
\end{gathered}
$$

(III) Assume $\lambda<1$ and let $f, g$ denote the functions of Theorem 3.1. Let

$$
\begin{gathered}
G=g+h ; \\
F=f \exp \left(h^{\prime}\right) .
\end{gathered}
$$

Then as $k, n \rightarrow \infty$ with (3.3) holding, we have

$$
\begin{gathered}
\frac{1}{n(1-\lambda)} \log w_{n, k} \rightarrow G(d) ; \\
\frac{w_{n, k}}{w_{n, k+1}} \rightarrow e^{G^{\prime}(d)}=F(d) .
\end{gathered}
$$

(IV) Assume $\lambda<1$ and let

$$
\omega^{*}=\frac{\omega-\lambda \delta_{0}}{1-\lambda} .
$$

The function $F$ is strictly decreasing on $(0,1)$ with range $(0, \infty)$. Its inverse $F^{-1}$ is related to $\omega^{*}$ by

$$
F^{-1}(x)=\int_{-A}^{0} \frac{y}{y-x} d \omega^{*}(y), x \in(0, \infty) .
$$

Moreover,

$$
G(d)=-(1-d) \log F(d)+\int_{-A}^{0} \log [F(d)-y] d \omega^{*}(y), d \in(0,1) .
$$


One can place hypotheses on the ratio of successive $\Delta_{n, j}$, instead of (3.11):

\section{Theorem 3.3}

Assume the hypotheses of Theorem 3.2, except that instead of (3.11), we assume that as $k, n \rightarrow \infty$ with (3.3) holding, we have,

$$
\frac{\Delta_{n, k}}{\Delta_{n, k+1}} \rightarrow J(d) .
$$

Instead of (3.16), define $F$ by

$$
F=f J
$$

and instead of (3.15), define $G$ by

$$
G=g+\int \log J
$$

Then all the conclusions of Theorem 3.2 persist.

We note that there is an extensive literature on zeros of composite polynomials, especially as regarding location of zeros [5], [6], [8], but the above are probably the first results on their zero distribution.

\section{Proof of Theorems $3.1,3.2$ and 3.3}

Throughout we assume the hypotheses of Theorem 3.1. The greatest integer $\leq x$ is denoted by $[x]$. We begin with

\section{Lemma 4.1}

(a) For $0<d<\lambda$,

$$
\left(\max _{k \leq d n} v_{n, k}\right)^{1 / n} \rightarrow 0 \text { as } n \rightarrow \infty .
$$

(b) For $d>\lambda$, there exists $n_{0}$ and a constant $C=C(d)>0$ with

$$
\left(\min _{k \geq d n} v_{n, k}\right)^{1 / n} \geq C, n \geq n_{0} .
$$

\section{Proof}

(a) Let $\varepsilon \in(0,1), d<\lambda^{\prime}<\lambda$. As $\nu$ has a point mass of size $\lambda$ at 0 , there exists $n_{0}=n_{0}(\varepsilon)$ such that for $n \geq n_{0}, V_{n}$ has $\geq \lambda^{\prime} n$ zeros in $[-\varepsilon, 0)$. From the definition of the coefficients in $V_{n}$,

$$
v_{n, k}=(-1)^{n-k} \sum_{1 \leq j_{1}<j_{2}<\ldots<j_{n-k} \leq n} \nu_{n, j_{1}} \nu_{n, j_{2}} \ldots \nu_{n, j_{n-k}} .
$$

Here all the $\nu_{n, j}$ lie in $[-A, 0)$, while all $v_{n, k}>0$. If $k \leq d n\left(<\lambda^{\prime} n\right)$, then by the pidgeonhole principle, at least $(n-k)-\left(n-\left[\lambda^{\prime} n\right]\right)=\left[\lambda^{\prime} n\right]-k$ of the terms in the products in $(4.3)$ must lie in $[0, \varepsilon]$. Hence

$$
\begin{aligned}
v_{n, k} & \leq \sum_{1 \leq j_{1}<j_{2}<\ldots<j_{n-k} \leq n} \varepsilon^{\left[\lambda^{\prime} n\right]-k} A^{n-\left[\lambda^{\prime} n\right]} \\
& =\varepsilon^{\left[\lambda^{\prime} n\right]-k} A^{n-\left[\lambda^{\prime} n\right]}\left(\begin{array}{c}
n \\
n-k
\end{array}\right) \\
& \leq \varepsilon^{\left[\lambda^{\prime} n\right]-[d n]}(2 A)^{n} .
\end{aligned}
$$


Then

$$
\limsup _{n \rightarrow \infty}\left(\max _{k \leq d n} v_{n, k}\right)^{1 / n} \leq \varepsilon^{\lambda^{\prime}-d} 2 A .
$$

Here the left-hand side, $d$ and $\lambda^{\prime}$ are independent of $\varepsilon$, and so we may let $\varepsilon \rightarrow 0+$ to get the result.

(b) Let $\lambda^{\prime \prime}>\lambda$. Then there exists $\varepsilon_{0} \in(0,1)$ such that

$$
\nu\left[0, \varepsilon_{0}\right]<\lambda^{\prime \prime}
$$

and hence for some $0<\varepsilon_{1}<\varepsilon_{0}$ and $n_{0}$, we have for $n \geq n_{0}$,

$$
\nu_{n}\left[0, \varepsilon_{1}\right] \leq \lambda^{\prime \prime} \text {. }
$$

If $k>\lambda^{\prime \prime} n$, we see that at least one of the terms in the sum in (4.3) has all $\left|\nu_{n, j_{i}}\right| \geq \varepsilon_{0}$. For example the term with the $n-k$ largest zeros (in absolute value) satisfies this. Then

$$
\min _{k \geq d n} v_{n, k} \geq \varepsilon_{0}^{n-k} \geq \varepsilon_{0}^{n}
$$

so (4.2) follows.

Next, we record a classic:

\section{Lemma 4.2}

If

$$
R(x)=\sum_{j=0}^{n} r_{j} x^{j}
$$

is a polynomial with all real zeros, then

$$
r_{j+1} r_{j-1} \leq \frac{j}{j+1} r_{j}^{2}
$$

In particular, if all $r_{j-1} r_{j+1}>0$, then $\left|\frac{r_{j+1}}{r_{j}}\right|$ decreases as $j$ increases.

Proof

See for example [8, p. 337, proof of Lemma 3].

\section{Lemma 4.3}

(a) For $0<d<\lambda$

$$
\max _{k \leq d n} \frac{v_{n, k}}{v_{n, k+1}} \rightarrow 0 \text { as } n \rightarrow \infty
$$

(b) For $d>\lambda$, there exists $n_{0}$ and a constant $C=C(d)>0$ with

$$
\min _{k \geq d n} \frac{v_{n, k}}{v_{n, k+1}} \geq C, n \geq n_{0} \text {. }
$$

Proof

(a) By Lemma 4.2, and the fact that $V_{n}$ is monic,

$$
\begin{aligned}
\frac{1}{v_{n, k}} & =\frac{v_{n, n}}{v_{n, k}} \\
& =\prod_{\ell=k}^{n-1} \frac{v_{n, \ell+1}}{v_{n, \ell}} \leq\left(\frac{v_{n, k+1}}{v_{n, k}}\right)^{n-k} .
\end{aligned}
$$


Hence

$$
-\log v_{n, k}^{1 / n} \leq \frac{n-k}{n} \log \frac{v_{n, k+1}}{v_{n, k}} .
$$

By Lemma 4.1, the left-hand side tends to $\infty$ uniformly for $k \leq d n$, and so the same is true of the right-hand side. Since $n-k \geq n(1-d)$ and $1-d>0$, we deduce (4.4).

(b) Suppose $d>\lambda^{\prime}>\lambda$. Let $j=j(n)=\left[\lambda^{\prime} n\right]$. Then Lemma 4.2 gives for $k \geq d n$,

$$
\frac{v_{n, k+1}}{v_{n, j}}=\prod_{\ell=j}^{k} \frac{v_{n, \ell+1}}{v_{n, \ell}} \geq\left(\frac{v_{n, k+1}}{v_{n, k}}\right)^{k+1-j}
$$

SO

$$
\log v_{n, k+1}^{1 / n}-\log v_{n, j}^{1 / n} \geq \frac{k+1-j}{n} \log \frac{v_{n, k+1}}{v_{n, k}} .
$$

Here by Lemma 4.1(b),

$$
v_{n, j}^{1 / n}=v_{n,\left[\lambda^{\prime} n\right]}^{1 / n} \geq C
$$

while we have from (4.3) the trivial bound

$$
v_{n, k+1} \leq A^{n-k-1}\left(\begin{array}{c}
n \\
n-k-1
\end{array}\right) \leq(2 A)^{n} .
$$

Since

$$
k-j \geq\left(d-\lambda^{\prime}\right) n,
$$

we deduce that

$$
\max _{k \geq d n} \log \frac{v_{n, k+1}}{v_{n, k}}
$$

is bounded above independent of $n$, and then (4.5) follows.

The next step is to factor out that part of $V_{n}$ with the small zeros. Choose $0<m_{n} \leq \lambda n$ such that

$$
\lim _{n \rightarrow \infty} m_{n} / n=\lambda
$$

and the smallest $m_{n}$ zeros of $V_{n}$ approach 0 as $n \rightarrow \infty$. More precisely, if we order the absolute values of the zeros of $V_{n}$ in increasing order, we are choosing $m_{n}$ such that

$$
\varepsilon_{n}:=\left|\nu_{n, m_{n}}\right| \rightarrow 0 \text { as } n \rightarrow \infty .
$$

This is possible, because $\nu$ has a mass point of size $\lambda$ at 0 . Next, write

$$
V_{n}(x)=V_{n}^{*}(x) S_{n}(x)
$$

where $S_{n}$ has degree $m_{n}$, is monic, and has as its zeros the $m_{n}$ smallest zeros (in absolute value) of $V_{n}$. Write

$$
V_{n}^{*}(x)=\sum_{j=0}^{n-m_{n}} v_{n, j}^{*} x^{j} .
$$

Note that all zeros of $V_{n}^{*}$ are non-positive, so all $v_{n, j}^{*} \geq 0$. 


\section{Lemma 4.4}

Let $r \geq 0$ and $\eta>0$.

(a) As $n \rightarrow \infty$,

$$
\sup _{k \geq m_{n}+\eta n}\left(\frac{1}{n} \log v_{n, k}-\frac{1}{n} \log v_{n, k-m_{n}}^{*}\right) \rightarrow 0 .
$$

(b) As $n \rightarrow \infty$, if $k=k(n) \geq m_{n}+\eta n$,

$$
\begin{aligned}
& \left|\frac{v_{n, k}}{v_{n, k+1}}-r\right| \\
\leq & o(1)+\max \left\{\left|\frac{v_{n, k-m_{n}}^{*}}{v_{n, k+1-m_{n}}^{*}}-r\right|,\left|\frac{v_{n, k-m_{n}+[\eta n]}^{*}}{v_{n, k+1-m_{n}+[\eta n]}^{*}}-r\right|\right\} .
\end{aligned}
$$

\section{Proof}

(a) Write

$$
S_{n}(x)=\sum_{j=0}^{m_{n}} s_{n, j} x^{j} .
$$

Since $S_{n}$ has all its zeros in $\left[-\varepsilon_{n}, 0\right)$, we see from the obvious analogue of (4.3) that

$$
s_{n, j} \leq\left(\begin{array}{c}
m_{n} \\
m_{n}-j
\end{array}\right) \varepsilon_{n}^{m_{n}-j}
$$

Next, for $k \geq m_{n}$,

$$
v_{n, k}=\sum_{j=0}^{m_{n}} s_{n, j} v_{n, k-j}^{*}
$$

while Lemma 4.2 applied to $V_{n}^{*}$ implies that for $0 \leq j \leq m_{n}$,

$$
v_{n, k-j}^{*} \leq v_{n, k-m_{n}}^{*}\left(\frac{v_{n, k-m_{n}+1}^{*}}{v_{n, k-m_{n}}^{*}}\right)^{m_{n}-j} .
$$

Since $s_{n, m_{n}}=1$, and all $s_{n, j} \geq 0, v_{n, k-j}^{*} \geq 0$, we deduce that

$$
\begin{aligned}
v_{n, k-m_{n}}^{*} & \leq v_{n, k} \leq v_{n, k-m_{n}}^{*} \sum_{j=0}^{m_{n}}\left(\begin{array}{c}
m_{n} \\
m_{n}-j
\end{array}\right)\left(\varepsilon_{n} \frac{v_{n, k-m_{n}+1}^{*}}{v_{n, k-m_{n}}^{*}}\right)^{m_{n}-j} \\
& =v_{n, k-m_{n}}^{*}\left(1+\varepsilon_{n} \frac{v_{n, k-m_{n}+1}^{*}}{v_{n, k-m_{n}}^{*}}\right)^{m_{n}} .
\end{aligned}
$$

Then

$$
\begin{aligned}
0 & \leq \frac{1}{n} \log v_{n, k}-\frac{1}{n} \log v_{n, k-m_{n}}^{*} \\
& \leq \frac{m_{n}}{n} \log \left(1+\varepsilon_{n} \frac{v_{n, k-m_{n}+1}^{*}}{v_{n, k-m_{n}}^{*}}\right) \\
& \leq \lambda \varepsilon_{n} \frac{v_{n, k-m_{n}+1}^{*}}{v_{n, k-m_{n}}^{*}} \\
& \leq \lambda \varepsilon_{n} \sup _{j \geq m_{n}+\eta n} \frac{v_{n, j-m_{n}+1}^{*}}{v_{n, j-m_{n}}^{*}},
\end{aligned}
$$


recall (4.6). Since in the sup, $\frac{j-m_{n}}{n-m_{n}} \geq \frac{\eta n}{n-m_{n}} \rightarrow \frac{\eta}{1-\lambda}>0$, Lemma 4.3(b) applied to the polynomials $\left\{V_{n}^{*}\right\}$ (for which $\nu^{*}$ does not have a mass point at 0 ) shows that the sup in the last right-hand side remains bounded as $n \rightarrow \infty$. Then (4.10) follows as $\varepsilon_{n} \rightarrow 0$ as $n \rightarrow \infty$.

(b) Let $\eta \in(0, \lambda)$. We have

$$
\begin{aligned}
& v_{n, k}-r v_{n, k+1} \\
= & \left(\sum_{j=0}^{m_{n}-[\eta n]-1}+\sum_{j=m_{n}-[\eta n]}^{m_{n}}\right) s_{n, j}\left(v_{n, k-j}^{*}-r v_{n, k+1-j}^{*}\right) . \\
= & : \Sigma_{1}+\Sigma_{2} .
\end{aligned}
$$

Now in $\Sigma_{1}$, we have $j<m n-\eta n$, and by (4.12),

$$
s_{n, j} \leq 2^{m_{n}} \varepsilon_{n}^{m_{n}-j} .
$$

Also

$$
\begin{aligned}
& \left|v_{n, k-j}^{*}-r v_{n, k+1-j}^{*}\right| / v_{n, k-m_{n}}^{*} \\
\leq & \left(\frac{v_{n, k-m_{n}+1}^{*}}{v_{n, k-m_{n}}^{*}}\right)^{m_{n}-j}+r\left(\frac{v_{n, k-m_{n}+1}^{*}}{v_{n, k-m_{n}}^{*}}\right)^{m_{n}-j+1} \\
\leq & C^{m_{n}-j+1}(1+r),
\end{aligned}
$$

where $C$ does not depend on $k, n, j$, but only on $\eta$. Here, as in (a), we are using Lemma 4.3(b) applied to the polynomials $\left\{V_{n}^{*}\right\}$. Then for large enough $n$,

$$
\begin{aligned}
\Sigma_{1} & \leq 2^{m_{n}}(1+r) C \sum_{j=0}^{m_{n}-[\eta n]-1}\left(C \varepsilon_{n}\right)^{m_{n}-j} \\
& \leq 2^{\lambda n}(1+r) C\left(C \varepsilon_{n}\right)^{[\eta n]}
\end{aligned}
$$

and in particular,

$$
\Sigma_{1}^{1 / n}=o(1)=o\left(v_{n, k-1}^{1 / n}\right)
$$

in view of Lemma 4.1(b). Then also

$$
\Sigma_{1}=o\left(v_{n, k-1}\right) \text {. }
$$

Next,

$$
\begin{aligned}
& \leq \quad\left|\Sigma_{2}\right| \\
& \leq \sup _{m_{n} \geq j \geq m_{n}-\eta n}\left|\frac{v_{n, k-j}^{*}}{v_{n, k+1-j}^{*}}-r\right| \sum_{j=m_{n}-[\eta n]}^{m_{n}} s_{n, j} v_{n, k+1-j}^{*} \\
& \leq \quad v_{n, k+1} \sup _{m_{n} \geq j \geq m_{n}-[\eta n]}\left|\frac{v_{n, k-j}^{*}}{v_{n, k+1-j}^{*}}-r\right|
\end{aligned}
$$

recall (4.13). By Lemma 4.2, $\frac{v_{n, k-j}^{*}}{v_{n, k+1-j}^{*}}$ is monotone in $j$, so

$$
\left|\Sigma_{2}\right| \leq v_{n, k+1} \max \left\{\left|\frac{v_{n, k-m_{n}}^{*}}{v_{n, k-m_{n}+1}^{*}}-r\right|,\left|\frac{v_{n, k-m_{n}+[\eta n]}^{*}}{v_{n, k-m_{n}+[\eta n]+1}^{*}}-r\right|\right\} .
$$

Together with (4.14), this gives the result. 
Next, we apply the result of Van-Assche, Fano and Ortolani to the polynomials $\left\{V_{n}^{*}\right\}$ :

\section{Lemma 4.5}

There is a concave and differentiable function $g:(0,1) \rightarrow \mathbb{R}$ and a function $f$ defined by $f=e^{g^{\prime}}$ with the following properties: as $j, n \rightarrow \infty$ with $\frac{j}{n-m_{n}} \rightarrow c \in(0,1)$, we have

$$
\begin{aligned}
& \frac{1}{n-m_{n}} \log v_{n, j}^{*} \rightarrow g(1-c) ; \\
& \frac{v_{n, j}^{*}}{v_{n, j+1}^{*}} \rightarrow e^{g^{\prime}(1-c)}=f(1-c) .
\end{aligned}
$$

The function $f$ is strictly decreasing on $(0,1)$ with range $(0, \infty)$. Its inverse $f^{-1}$ is related to the measure

$$
\nu^{*}=\frac{\nu-\lambda \delta_{0}}{1-\lambda}
$$

by

$$
f^{-1}(x)=\int_{-A}^{0} \frac{y}{y-x} d \nu^{*}(y), x \in(0, \infty)
$$

Moreover,

$$
g(d)=-(1-d) \log f(d)+\int_{-A}^{0} \log [f(d)-y] d \nu^{*}(y), d \in(0,1) .
$$

\section{Proof}

Let $\nu_{n}^{*}$ denote the zero counting measure of $V_{n}^{*}$. Since we formed $V_{n}^{*}$ from $V_{n}$ by removing the $m_{n}$ zeros of $S_{n}$, it follows that

$$
\nu_{n}^{*}=\frac{1}{n-m_{n}}\left(n \nu_{n}-m_{n} \sigma_{n}\right),
$$

where $\sigma_{n}$ denotes the zero counting measure of $S_{n}$. Then clearly

$$
\nu_{n}^{*} \rightarrow \frac{\nu-\lambda \delta_{0}}{1-\lambda}=\nu^{*} .
$$

Since $\nu^{*}$ does not have a mass point at 0 , the result now follows from the classical result of Van Assche, Fano and Ortolani [1, Theorem 1, p. 1598]. We note that $V_{n}^{*}$ has degree $n-m_{n}$ and that in [1, Theorem 1, p. 1598], the coefficients are indexed in a reverse order to (4.9). Moreover, we also use that as

$$
\frac{j}{n-m_{n}} \rightarrow c, \text { so } \frac{n-m_{n}-j}{n-m_{n}} \rightarrow 1-c .
$$

Proof of Theorem 3.1

(I) This follows from Lemma 4.1 and 4.3.

(II) Suppose $k, n \rightarrow \infty$ with

$$
\frac{k}{n} \rightarrow 1-(1-\lambda) d \in(\lambda, 1) .
$$


Then $j=k-m_{n}$ satisfies

$$
\begin{aligned}
\frac{j}{n-m_{n}} & =\frac{k / n-m_{n} / n}{1-m_{n} / n} \\
& \rightarrow \frac{1-(1-\lambda) d-\lambda}{1-\lambda}=1-d .
\end{aligned}
$$

From Lemma 4.4 and then Lemma 4.5, as $n \rightarrow \infty$,

$$
\begin{aligned}
\frac{1}{n(1-\lambda)} \log v_{n, k} & =(1+o(1)) \frac{1}{n-m_{n}} \log v_{n, k-m_{n}}^{*}+o(1) \\
& =(1+o(1)) \frac{1}{n-m_{n}} \log v_{n, j}^{*}+o(1) \\
& =g(d)+o(1) .
\end{aligned}
$$

Next, from Lemma 4.4, given a real number $r$,

$$
\begin{aligned}
& \left|\frac{v_{n, k}}{v_{n, k+1}}-r\right| \\
\leq & o(1)+\max \left\{\left|\frac{v_{n, k-m_{n}}^{*}}{v_{n, k-m_{n}+1}^{*}}-r\right|,\left|\frac{v_{n, k-m_{n}+[\eta n]}^{*}}{v_{n, k-m_{n}+[\eta n]+1}^{*}}-r\right|\right\} .
\end{aligned}
$$

Here if (4.20) holds, we have by (4.16) of Lemma 4.5

and

$$
\frac{v_{n, k-m_{n}}^{*}}{v_{n, k-m_{n}+1}^{*}} \rightarrow f(d)
$$

$$
\frac{v_{n, k-m_{n}+[\eta n]}^{*}}{v_{n, k-m_{n}+[\eta n]+1}^{*}} \rightarrow f\left(d-\frac{\eta}{1-\lambda}\right),
$$

so choosing $r=f(d)$ above,

$$
\begin{aligned}
& \left|\frac{v_{n, k}}{v_{n, k+1}}-f(d)\right| \\
\leq & o(1)+\left|f\left(d-\frac{\eta}{1-\lambda}\right)-f(d)\right| .
\end{aligned}
$$

Now from (4.18), it is clear that the inverse $f^{-1}$ of $f$ is continuous, so the same is true of $f$. Then as the left-hand side is independent of $\eta$, we can let $\eta \rightarrow 0+$ to deduce

(III) This follows from Lemma 4.5.

$$
\frac{v_{n, k}}{v_{n, k+1}} \rightarrow f(d)
$$

\section{Proof of Theorem 3.2}

We first show that any weak subsequential limit $\omega$ of $\left\{\omega_{n}\right\}$ has a mass point of size exactly $\lambda$ at 0 . Observe that

$$
\frac{w_{n, k}}{w_{n, k+1}} / \frac{v_{n, k}}{v_{n, k+1}}=\frac{\Delta_{n, k}}{\Delta_{n, k+1}}
$$

and let $\eta \in\left(0, \frac{1}{2}\right)$. From our hypothesis (3.12) on $\left\{\Delta_{n, j}\right\}$ there exists $C>1$ such that

$$
\frac{1}{C} \leq \frac{w_{n, k}}{w_{n, k+1}} / \frac{v_{n, k}}{v_{n, k+1}} \leq C, \text { when } k / n \in(\eta, 1-\eta) .
$$


By Lemma 4.2, $w_{n, k} / w_{n, k+1}$ is a monotone function of $k$, so it follows from Lemma 4.3(a), that if $d<\lambda$,

$$
\sup _{k \leq d n} \frac{w_{n, k}}{w_{n, k+1}} \rightarrow 0 \text { as } n \rightarrow \infty .
$$

Similarly that lemma gives if $d>\lambda$, then for some $n_{0}$ and $n \geq n_{0}$,

$$
\inf _{k \geq d n} \frac{w_{n, k}}{w_{n, k+1}} \geq C>0
$$

Now choose an infinite subsequence of integers $S$ such that as $n \rightarrow \infty$ through $S$, the zero counting measures $\left\{\omega_{n}\right\}$ of $W_{n}$ converge weakly to some measure $\omega$. Assume $\omega$ has a mass point of size $\lambda_{1}$ at 0 . We may apply Theorem 3.1 (or Lemmas 4.1 and 4.3) to this subsequence $\left\{W_{n}\right\}_{n \in S}$. Those results and (4.22), (4.23) show that necessarily $\lambda_{1}=\lambda$. Next, Theorem 3.1 applied to this subsequence, provides a concave function $G$ such that as $n \rightarrow \infty$ through $S$ with (4.20) holding, we have

$$
\begin{gathered}
\frac{1}{n(1-\lambda)} \log w_{n, k} \rightarrow G(d) ; \\
\frac{w_{n, k}}{w_{n, k+1}} \rightarrow e^{G^{\prime}(d)}=F(d) .
\end{gathered}
$$

Moreover if

$$
\omega^{*}=\frac{\omega-\lambda \delta_{0}}{1-\lambda},
$$

then

$$
\begin{gathered}
F^{-1}(x)=\int_{-A}^{0} \frac{y}{y-x} d \omega^{*}(y), x \in(0, \infty) \\
G(d)=-(1-d) \log F(d)+\int_{-A}^{0} \log [F(d)-y] d \omega^{*}(y), d \in(0,1) .
\end{gathered}
$$

Now by our hypotheses (3.10) and (3.11), and by Theorem 3.1, as $k, n \rightarrow \infty$, with (3.3) holding, we have

$$
\begin{aligned}
& \frac{1}{n(1-\lambda)} \log w_{n, k} \\
= & \frac{1}{n(1-\lambda)} \log v_{n, k}+\frac{1}{n(1-\lambda)} \log \Delta_{n, k} \\
\rightarrow & g(d)+h(d) .
\end{aligned}
$$

Then the function $G$ arising above from $\left\{\omega_{n}\right\}_{n \in S}$ satisfies

$$
G=g+h
$$

and so is independent of the subsequence. Hence the function $F$ arising above from $\left\{\omega_{n}\right\}_{n \in S}$ must also be independent of the subsequence, and

$$
\log F=G^{\prime}=g^{\prime}+h^{\prime} .
$$


Note here that $h=G-g$ must be differentiable. Now we prove that $\omega$ is independent of the subsequence. Suppose that $\omega$ and $\omega^{1}$ are weak limits of different subsequences of $\left\{\omega_{n}\right\}$. Since $F$ above is independent of the subsequence, we obtain

$$
\int_{-A}^{0} \frac{y}{y-x} d\left(\omega-\omega^{1}\right)(y)=0, x \in(0, \infty) .
$$

For $z \in \mathbb{C} \backslash[-A, 0]$, define

$$
\Psi(z)=\int_{-A}^{0} \frac{y}{y-z} d\left(\omega-\omega^{1}\right)(y) .
$$

We see that $\Psi=0$ in $(0, \infty)$, and hence as it is analytic outside $[-A, 0]$, we deduce that $\Psi$ is identically 0 . Finally, the Perron-Stieltjes inversion formula gives for each $[a, b] \subset(-A, 0)$,

$$
0=\lim _{\varepsilon \rightarrow 0+} \frac{1}{\pi} \int_{a}^{b} \operatorname{Im} \Psi(x+i \varepsilon) d x=\left(\omega-\omega^{1}\right)([a, b]) .
$$

Then the uniqueness follows. Thus we have established (I), (II), (III), while (IV) follows from (4.26-7).

\section{Proof of Theorem 3.3}

Here by hypothesis, and (4.25), as $k / n \rightarrow \infty$ with (3.3) holding, we have

$$
\begin{aligned}
F(d) & =\lim \frac{w_{n, k}}{w_{n, k+1}} \\
& =\lim \frac{v_{n, k}}{v_{n, k+1}} \frac{\Delta_{n, k}}{\Delta_{n, k+1}}=f(d) J(d) .
\end{aligned}
$$

That is $F=f J$, and, taking logs,

$$
G^{\prime}=g^{\prime}+\log J
$$

\section{Proof of the Results of Sections 1 and 2}

From Proposition 1.1,

$$
(-4 n)^{-n} P_{n}(-4 n z)=\sum_{j=0}^{n} p_{n, j} z^{j}=\sum_{j=0}^{n} \Delta_{n, j} q_{n, j} z^{j},
$$

where

$$
\Delta_{n, j}=\frac{\Gamma(\alpha+n+1)}{\Gamma(\alpha+j+1)}(4 n)^{j-n} .
$$

\section{Lemma 5.1}

Assume the hypotheses of Theorem 1.2.

(a)

$$
\frac{\Delta_{n, k}}{\Delta_{n, k+1}}=\frac{\alpha+k+1}{4 n} .
$$

(b) Let $d, \lambda \geq 0$. As $k, n \rightarrow \infty$ with $k / n \rightarrow 1-(1-\lambda) d \in(\lambda, 1)$,

$$
\frac{\Delta_{n, k}}{\Delta_{n, k+1}} \rightarrow \frac{1-(1-\lambda) d}{4} .
$$


(c) All zeros of $P_{n}$ lie in the interval $\left(0,\left(4 n+2 \alpha+2 \frac{1}{2}\right) / B\right)$.

Proof

(a), (b) These are immediate.

(c) We use a classical theorem of Szegö [2, p. 23], [12, p. 66], [13, p. 191]. If $R$ has all its zeros in $|z|<r$ and $S$ has all its zeros in $|z| \leq s$, then $R \# S$ has all its zeros in $|z| \leq r s$. In our case $P_{n}$ is a constant multiple of $Q_{n} \# L_{n}^{(\alpha)}$. Here $Q_{n}$ has all its zeros in $[-1 / B, 0)$ while all zeros of $L_{n}^{(\alpha)}$ are positive, with the largest zero bounded above by [22, p.128]

$$
\begin{aligned}
& 2 n+\alpha+1+\left\{(2 n+\alpha+1)^{2}+\frac{1}{4}-\alpha^{2}\right\}^{1 / 2} \\
< & 4 n+2 \alpha+2 \frac{1}{2} .
\end{aligned}
$$

Then all zeros of $P_{n}$ lie in the disk $|z|<\left(4 n+2 \alpha+2 \frac{1}{2}\right) / B$. Since all zeros of $P_{n}$ are positive, we obtain the result.

\section{Proof of Theorem 1.2}

Assume first that $\nu_{n} \stackrel{*}{\rightarrow} \nu$ as $n \rightarrow \infty$. It follows from the lemma above that the zero counting measure $\mu_{n}$ of $P_{n}(-4 n x)$ has support in $\left(-\left(1+\frac{\left(2 \alpha+2 \frac{1}{2}\right)}{4 n}\right) / B, 0\right)$. Moreover, (5.4) above shows that as $k, n \rightarrow \infty$ with (3.3) holding,

$$
\frac{\Delta_{n, k}}{\Delta_{n, k+1}} \rightarrow J(d)=\frac{1-(1-\lambda) d}{4}
$$

Then all the hypotheses of Theorem 3.3 hold, with $W_{n}$ and $V_{n}$ taken as $P_{n}$ and $Q_{n}$, respectively. By Theorem 3.3, $\mu_{n} \stackrel{*}{\rightarrow} \mu$ as $n \rightarrow \infty$, and $\mu$ will have a mass point at 0 of size $\lambda$ if $\nu$ does.

Conversely, if we assume that $\mu_{n} \stackrel{*}{\rightarrow} \mu$ as $n \rightarrow \infty$, then we can apply Theorem 3.3 with the roles of $P_{n}(-4 n x)$ and $Q_{n}$ reversed. Indeed, with the notation (5.1), we have

$$
Q_{n}(x)=\sum_{j=0}^{n} p_{n, j} \Delta_{n, j}^{-1} x^{j} .
$$

Here $\Delta_{n, j}^{-1}$ satisfies similar hypotheses to those of $\Delta_{n, j}$.

\section{Proof of Theorems 1.3 and 1.4}

Taking $V_{n}=Q_{n}$ in Theorem 3.1, we obtain (1.11), (1.12), (1.16), (1.17), (1.21), and (1.22). Theorem 3.3 gives (1.13), (1.14), (1.20), (1.21), and (1.23). Next, in Theorem 3.3, we may take

$$
F(d)=f(d) J(d)=f(d) \frac{1-(1-\lambda) d}{4} .
$$

Then

$$
\begin{aligned}
G(x) & =g(x)+\int \log J(x) d x \\
& =g(x)-x \ln 4-\frac{1}{1-\lambda}\{(1-(1-\lambda) x) \log (1-(1-\lambda) x)-(1-(1-\lambda) x)\} .
\end{aligned}
$$




\section{Proof of Theorem 1.5}

(I) Cauchy's integral formula and (1.6) give

$$
\begin{aligned}
\frac{(-1)^{n}}{\Gamma(n+\alpha+1)} P_{n}(x) & =\sum_{j=0}^{n} \frac{(-x)^{j}}{\Gamma(\alpha+j+1)} \frac{1}{2 \pi i} \int_{|t|=1} \frac{Q_{n}(t)}{t^{j+1}} d t \\
& =\frac{1}{2 \pi i} \int_{|t|=1} \frac{Q_{n}(t)}{t} \sum_{j=0}^{n} \frac{(-x / t)^{j}}{\Gamma(\alpha+j+1)} d t .
\end{aligned}
$$

Since

$$
\frac{1}{2 \pi i} \int_{|t|=1} \frac{Q_{n}(t)}{t^{n+k}} d t=0, k \geq 2
$$

we can then replace the partial sum

$$
\sum_{j=0}^{n} \frac{(-x / t)^{j}}{\Gamma(\alpha+j+1)}=\frac{1}{\Gamma(\alpha+1)} \sum_{j=0}^{n} \frac{(-x / t)^{j}}{(\alpha+1)_{j}}
$$

of $\frac{1}{\Gamma(\alpha+1)}{ }_{1} F_{1}\left(1 ; \alpha+1 ;-\frac{x}{t}\right)$ by the complete series, taking also account of its entirety.

(II) This follows easily from Proposition 1.1.

(III) As in the proof of Proposition 1.1,

$$
\frac{(-1)^{n}}{\Gamma(\alpha+n+1)} \int_{0}^{\infty} P_{n}(x) e^{-s x} x^{\alpha} d x=s^{-\alpha-1} Q_{n}\left(-s^{-1}\right),
$$

that is, $s^{-\alpha-1} Q_{n}\left(-s^{-1}\right)$ is the Laplace transform of $\frac{(-1)^{n}}{\Gamma(\alpha+n+1)} x^{\alpha} P_{n}(x)$. Then (1.27) follows by the standard inversion formula for Laplace transforms.

(IV) Recall our abbreviation $\sigma_{k}=\sigma_{n, k}$. Let

$$
\omega_{j, k}=\int_{0}^{\infty} x^{j+\alpha} e^{-\sigma_{k} x} d x=\sigma_{k}^{-(\alpha+j+1)} \Gamma(j+\alpha+1) .
$$

Then by integrating, we see that

$$
P_{n}^{\#}(x)=\operatorname{det}\left[\begin{array}{ccccc}
\omega_{0,1} & \omega_{1,1} & \omega_{2,1} & \cdots & \omega_{n, 1} \\
\omega_{0,2} & \omega_{1,2} & \omega_{2,2} & \cdots & \omega_{n, 2} \\
\vdots & \vdots & \vdots & \ddots & \vdots \\
\omega_{0, n} & \omega_{1, n} & \omega_{2, n} & \cdots & \omega_{n, n} \\
1 & x & x^{2} & \cdots & x^{n}
\end{array}\right]
$$

satisfies the biorthogonality relations (1.1). Substituting in the formula for the moments $\omega_{j, k}$ and extracting a factor of $\sigma_{j}^{-(\alpha+2)}$ from the $j$ th row, and $\Gamma(\alpha+k)$ from the $k$ th column shows that $P_{n}^{\#}$ admits the representation (1.28), apart from a constant. By comparing leading coefficients, we see that the monic polynomial $P_{n}$ has the form (1.28). Of course, the denominator determinant in (1.28) is a Vandermonde determinant, and so is non-vanishing.

\section{Proof of Corollary 2.1}


Here

$$
Q_{n}(y)=\prod_{k=1}^{n}\left(y+\frac{1}{k+\beta}\right)
$$

has all its zeros in $\left[-\frac{1}{1+\beta}, 0\right)$, so we may choose $B=1+\beta$ in Theorem 1.2. Lemma $5.1(\mathrm{c})$ shows that all zeros of $\Lambda_{n}^{(\alpha, \beta)}$ lie in $\left(0,\left(4 n+2 \alpha+2 \frac{1}{2}\right) /(1+\beta)\right)$. We have already noted that for this case $\nu_{n} \stackrel{*}{\rightarrow} \delta_{0}$ as $n \rightarrow \infty$. Theorem 1.2 shows that also $\mu_{n} \stackrel{*}{\rightarrow} \delta_{0}$ as $n \rightarrow \infty$.

\section{Proof of Corollary 2.2}

Write

$$
\begin{aligned}
(-1)^{n} G_{n}^{(\alpha)}(-4 n z) & =\sum_{j=0}^{n}\left(\begin{array}{l}
n \\
j
\end{array}\right) \frac{(j+1)^{n}}{\Gamma(j+\alpha+1)}(4 n z)^{j} \\
& =\sum_{j=0}^{n} g_{n, j} z^{j}
\end{aligned}
$$

Recall that $G_{n}$ is biorthogonal to the exponentials $\left\{e^{-\sigma_{n, k} x}\right\}_{k=1}^{n}$, where now $D_{n}^{(0,0)}\left(\sigma_{n, k}^{-1}\right)=$ 0 and

$$
D_{n}^{(0,0)}(t)=\sum_{j=0}^{n}(-1)^{n-j}\left(\begin{array}{l}
n \\
j
\end{array}\right)(j+1)^{n} t^{j} .
$$

Indeed this follows from Proposition 1.1 and the definitions of $G_{n}^{(\alpha)}$ and $D_{n}^{(0,0)}$, as well as a transformation $x \rightarrow-x$. Since all the zeros of $D_{n}^{(0,0)}$ lie in $(0,1)[9]$, so we may choose $B=1$ in Theorem 1.2. The authors proved in 1994 [9] that the zero counting measures of $D_{n}^{(0,0)}(-x)$ converge to an absolutely continuous measure $\nu^{\prime}(-x) d x$, where

$$
\nu^{\prime}(-x)=-\frac{1}{\pi h^{\prime}\left(h^{[-1]}(x)\right)}, x \in(0,1),
$$

and

$$
h(y)=\frac{\sin y}{y} e^{y \cot y-1}, y \in[0, \pi]
$$

is a strictly decreasing function. By Theorem 1.2, the zero counting measures of $G_{n}(-4 n x)$ converge weakly to a measure with support in $[-1,0]$, having no mass point at 0 . We can directly compute that as $k, n \rightarrow \infty$ in such a way that $k / n \rightarrow 1-d$

$$
\begin{aligned}
\frac{g_{n, k}}{g_{n, k+1}} & =\frac{k+1}{n-k}\left(1-\frac{1}{k+2}\right)^{n} \frac{k+\alpha+1}{4 n} \\
& \rightarrow \frac{(1-d)^{2}}{4 d} \exp \left(-\frac{1}{1-d}\right)=F(d)
\end{aligned}
$$

Note that

$$
G^{\prime}(d)=\ln F(d)=2 \ln (1-d)-\ln (4 d)-\frac{1}{1-d},
$$


SO

$$
\begin{aligned}
G(d) & =2[-(1-d) \ln (1-d)+(1-d)]-d \ln 4-d \ln d+d+\ln (1-d) \\
& =-2(1-d) \ln (1-d)-d \ln d-2 d \ln 2-d+\{2+\ln (1-d)\} .
\end{aligned}
$$

If we ignore the the term in brackets \{\} in the last line, we obtain the function that corresponds to zeros of Laguerre polynomials [1, p. 1612]. Moreover, if we examine

$$
\begin{aligned}
D_{n}^{(\alpha, \beta)}(t) & =\sum_{j=0}^{n}(-1)^{n-j}\left(\begin{array}{c}
n \\
j
\end{array}\right)(\beta+j+1)^{\alpha+n} t^{j} \\
& =\sum_{j=0}^{n}(-1)^{n-j} d_{n, j} t^{j}
\end{aligned}
$$

then we see that as $k, n \rightarrow \infty$ in such a way that $k / n \rightarrow 1-d$,

$$
\begin{aligned}
\frac{d_{n, k}}{d_{n, k+1}} & =\frac{k+1}{n-k}\left(1-\frac{1}{k+\beta+2}\right)^{n}(1+o(1)) \\
& \rightarrow \frac{1-d}{d} \exp \left(-\frac{1}{1-d}\right) .
\end{aligned}
$$

This is independent of $\alpha, \beta$, and since we know that for $\alpha=\beta=0$, the asymptotic zero distribution is given by (5.5-5.6), the same zero distribution holds for all $\alpha, \beta$.

\section{Proof of Corollary 2.3}

Write

$$
\begin{aligned}
(-1)^{n} M_{n}^{(\alpha)}(-4 n x) & =\sum_{j=0}^{n}\left(\begin{array}{l}
n \\
j
\end{array}\right) \frac{(j+1)_{n}}{\Gamma(j+\alpha+1)}(4 n x)^{j} \\
& =\sum_{j=0}^{n} m_{n, j} x^{j} .
\end{aligned}
$$

Recall that $M_{n}^{(\alpha)}$ is biorthogonal to the exponentials $\left\{e^{-\sigma_{n, k} x}\right\}_{k=1}^{n}$, where now $Q_{n}\left(-\sigma_{n, k}^{-1}\right)=0,1 \leq k \leq n$, and $Q_{n}(-x)$ is the Legendre polynomial for $[0,1]$. It is known that all zeros of $\left\{Q_{n}\right\}$ lie in $(0,1)$, and hence all zeros of $Q_{n}(-x)$ lie in $(-1,0)$. Thus we may choose $B=1$ in Theorem 1.2. Moreover [21], the zero counting measures for the Legendre polynomial on $[0,1]$ converge weakly to

$$
\frac{d x}{\pi \sqrt{x(1-x)}} \text { on }(0,1) \text {, }
$$

so a corresponding statement is true for $\left\{\nu_{n}\right\}$ on $[-1,0]$. By Theorem 1.2 , the $\left\{\mu_{n}\right\}$ converge weakly to a measure $\mu$ on $[-1,0]$ with no mass point at 0 . Moreover, if $n, k \rightarrow \infty$ with $k / n \rightarrow 1-d$,

$$
\begin{aligned}
\frac{m_{n, k}}{m_{n, k+1}} & =\frac{k+1}{n-k} \frac{k+1}{k+n+1} \frac{k+\alpha+1}{4 n} \\
& \rightarrow \frac{(1-d)^{3}}{4 d(2-d)}=F(d) .
\end{aligned}
$$




\section{REFERENCES}

[1] W. Van Assche, G. Fano and F. Ortolani, Asymptotic Behavior of the Coefficients of Some Sequences of Polynomials, SIAM J. Math Anal., 18(1987), 1597-1615.

[2] P. Borwein, T. Erdelyi, Polynomials and Polynomial Inequalities, Springer, New York, 1995.

[3] C. Brezinski, Biorthogonality and its Applications to Numerical Analysis, Marcel Dekker, New York, 1992.

[4] P. Henrici, Applied and Computational Complex Analysis, Vol. 1, Wiley, New York, 1974.

[5] A. Iserles and S.P. Norsett, Bi-orthogonality and Zeros of Transformed Polynomials, J. Comp. Appl. Math., 19(1987), 39-45.

[6] A. Iserles, S.P. Norsett, E.B. Saff, On Transformations and Zeros of Polynomials, Rocky Mountain J. Math., 21(1991), 331-357.

[7] A. Iserles and E.B. Saff, Zeros of expansions in orthogonal polynomials, Math. Proc. Camb. Phil. Soc., 105(1989), 559-573.

[8] B. Ja. Levin, Distribution of Zeros of Entire Functions, Translations of Math. Monographs, Vol. 5, American Mathematical Society, Providence, 1972.

[9] D.S. Lubinsky and A. Sidi, Strong Asymptotics for Polynomials Biorthogonal to Powers of $\log x$, Analysis, 14(1994), 341-379.

[10] D.S. Lubinsky and I. Soran, Weights whose Biorthogonal Polynomials admit a Rodrigues Formula, JMAA, 324(2006), 805-819.

[11] D.S. Lubinsky and H. Stahl, Some Explicit Biorthogonal Polynomials, (in) Approximation Theory XI (eds. C.K. Chui, M. Neamtu, L.L. Schumaker), Nashboro Press, Brentwood, TN, 2005, pp. 279-285.

[12] M. Marden, Geometry of Polynomials, American Mathematical Society, Providence, 1949.

[13] G.V. Milovanovic, D.S. Mitrinovic, and T.M. Rassias, Topics in Polynomials: Extremal Problems, Inequalities, Zeros, World Scientific, Singapore, 1994.

[14] E.D. Rainville, Special Functions, Chelsea, 1960.

[15] A. Sidi, Numerical Quadrature and Non-Linear Sequence Transformations: Unified Rules for Efficient Computation of Integrals with Algebraic and Logarithmic Endpoint Singularities, Math. Comp., 35(1980), 851-874.

[16] A. Sidi, Numerical quadrature for some infinite range integrals, Math. Comp., 38(1982), 127-142.

[17] A. Sidi, Problems 5-8, (in) Numerical Integration III, (eds. H. Brass and G. Hämmerlin), Birkhäuser, Berlin, 1988, pp. 321-325.

[18] A. Sidi, Practical Extrapolation Methods, Cambridge University Press, Cambridge, 2003.

[19] A. Sidi and D.S.Lubinsky, On the Zeros of Some Polynomials that Arise in Numerical Quadrature and Convergence Acceleration, SIAM J. Numer. Anal., 20(1983), 589-598.

[20] A. Sidi and D.S. Lubinsky, Biorthogonal Polynomials and Numerical Integration Formulas for Infinite Intervals, manuscript.

[21] H. Stahl and V. Totik, General Orthogonal Polynomials, Cambridge University Press, Cambridge, 1992.

[22] G. Szegő, Orthogonal Polynomials, 4th edn., American Mathematical Society Colloquium Publications, Vol. 23, American Math. Soc. Providence, 1975.

${ }^{1}$ School of Mathematics, Georgia Institute of Technology, Atlanta, GA 30332-0160, USA., Lubinsky@math.gatech.edu, ${ }^{2}$ Department of Computer Science, Technion-Israel Institute of Technology, Haifa 32000, Israel, Asidi@CS.technion.aC.IL 